\title{
A call for doctors to recommend antibiotic-free foods: agricultural antibiotics and the public health crisis of antimicrobial resistance
}

\author{
Martin A. Makary ${ }^{1,2}$ - Katerina Kaczmarski ${ }^{2} \cdot$ Keeve Nachman ${ }^{3,4}$
}

Received: 10 December 2017 / Revised: 6 March 2018 / Accepted: 29 March 2018 / Published online: 31 May 2018

(c) The Author(s) under exclusive licence to the Japan Antibiotics Research Association 2018

While it is widely known that antimicrobial resistance (AMR) is a consequence of antibiotic overuse in patients, the contribution of routine antibiotic use in animal agriculture to this emerging public health crisis is often overlooked. According to the $\mathrm{CDC}$, agricultural antibiotic use is responsible for $\sim 20 \%$ of resistant infections in humans. Just as physicians led an anti-smoking campaign to educate patients and communities, so too should we draw attention to the association between routine antibiotic use in animals and the declining efficacy of antibiotics in treating human infections. In this article, we highlight the health and economic consequences of antibiotic misuse in animal production and detail how physicians can use their role and influence to reduce AMR from agriculture.

Agricultural antibiotics became widespread more than a half century ago when AMR was not recognized to be a public health problem. At that time, antibiotics were discovered to accelerate pig and chicken growth when administered in conjunction with vitamins [1,2]. Since then, antimicrobial use in animal agriculture has become widespread, and sale of these drugs for veterinary use exceeds that for human use by nearly threefold. While organic animal production (which by definition does not include the use of antimicrobials) is increasing in the poultry sector, less than $1 \%$ of US farmland dedicated to livestock production is certified

Martin A. Makary

mmakary1@jhmi.edu

1 Department of Health Policy and Management, Johns Hopkins Bloomberg School of Public Health, Baltimore, Maryland, USA

2 Department of Surgery, Johns Hopkins University School of Medicine, Baltimore, Maryland, USA

3 Department of Environmental Health and Engineering, Johns Hopkins Bloomberg School of Public Health, Baltimore, Maryland, USA

4 Johns Hopkins Center for a Livable Future, Johns Hopkins University, Baltimore, Maryland, USA as organic, suggesting that the vast majority of meat, egg, and dairy products in the grocery store are raised with antibiotics [3]. Among the most commonly used agricultural antibiotics are tetracyclines, aminoglycosides, sulfas, and penicillins [4]. A February 2017 World Health Organization report declared AMR the leading public health threat of our time. The report named 12 antibiotic-resistant superbugs, including superbugs resistant to the above antibiotics commonly used in food production, which pose an enormous health and bioterrorism threat.

AMR is accelerated by modern industrialized animal production practices, in which large numbers of animals are raised in crowded and unhygienic spaces, which serve as ideal breeding grounds for the development, propagation, and spread of antibiotic-resistant bacteria and resistance genes. While the industry has repeatedly denied or downplayed the role of animal agriculture in resistant infections in humans [5], there are many documented routes by which these organisms can be transferred from farms to humans, including through the use of manure as fertilizer, food animal transport, non-domesticated animals, and agricultural workers [6]. Furthermore, routine agricultural use of antibiotics within the same class as those used in humans can result in cross resistance. For example, sparfloxacin use in animals can promote development of fluoroquinoloneresistant bacteria. One study found that within 4 years of initiating routine prophylactic enrofloxacin use in chicken production, $20 \%$ of sampled chicken breasts contained ciprofloxacin-resistant Campylobacter, an alarming prevalence that increased further to $30 \% 10$ years later [7]. Additional evidence pointing to poultry consumption as a pathway of human exposure and an associated increase in ciprofloxacin-resistant Campylobacter infections in humans led the Food and Drug Administration to ban the use of fluoroquinolones in chickens in 2005 [8]. After the ban, bacterial resistance to ciprofloxacin in chicken breast samples decreased to $13.5 \%$, demonstrating the effectiveness of discontinuing routine antibiotic use in reducing AMR. This intervention, however, targeted only one antibiotic class in 
one animal species, while agricultural use of other antibiotics remains widespread.

Despite pressure from the public and an array of public health societies, scarce progress has been made to change the way antibiotics are routinely used in food animal production. In the absence of major progress on this issue, physicians can play a lead role in advocating for an end to prophylactic use of antibiotics in animals and encourage food producers, restaurant chains, and grocery stores to offer animal products produced without antibiotics. Hospitals are among the largest purchasers of food in many communities; at a minimum, we should demand that our hospitals purchase animal products only from producers who do not use antibiotics for purposes other than treating sick animals. Second, the physician community should ask the FDA to consider additional restrictions on the use of antibiotics in food animal production. Finally, we should educate our patients about the health and societal benefits of only purchasing animal products bearing labels indicating responsible antibiotic use (e.g., USDA-certified Organic).

Physicians have a powerful track record in uniting to impact the food industry. We markedly changed the food industry by initiating consumer demand with the campaign to buy low-fat foods, which ultimately led to a broad awareness and consumer-conscious public. While the basis of the low-fat food campaign was not evidence-based and later suggested to be scientifically flawed, it demonstrated the societal impact of a consensus physician recommendation on consumer behavior. Doctors should routinely incorporate a recommendation of antibiotic-free foods into nutrition and lifestyle discussions with patients.

Advocacy works. Currently, the American Medical Association (AMA) and the American Public Health Association are launching a national effort to promote antibiotic-free animal production. Environmental groups are also increasing public awareness to move the food industry toward antimicrobial stewardship in production. Several national restaurants have responded to the movement, including Chipotle and Panera, and grocery stores such as Whole Foods, HEB Natural, and Central Market Natural, by offering animal products raised without antibiotics. In response to consumer demand, other grocery stores have expanded their selection of these products. Chipotle became an early disruptor in the food service industry when they introduced antibiotic-free pork, a change that was associated with a doubling of carnitas burrito sales. On the basis of this success, they transitioned all meat products to antibioticfree. At the same time, Perdue Farms, a Maryland-based meat production company, eliminated all non-therapeutic antibiotic use in their chickens, in part because of consumer concerns related to public health advocacy around antibiotics. Chick-Fil-A and Subway shortly thereafter pledged to transition to meats produced without antibiotics. Now, over half of the largest 25 fast food and fast casual restaurant chains in the US have adopted policies to limit or eliminate meat products produced with antibiotics. While these restaurant chains have not yet fulfilled this transition, informed consumers continue to fuel this trend in demand.

An industrial transition to antibiotic-free meat production has been shown to be economically plausible with little effect on wholesale meat prices. One study conducted by the USDA demonstrated a $<1 \%$ increase in wholesale meat prices and minimal decrease in farm productivity and output in response to industry-wide antibiotic-free production practices [9]. Despite this, antibiotic-free products remain in the minority on a national scale. As an economic result of its scarcity, they can sometimes be more expensive than conventional products. Further advocacy is needed to ensure that the health of poorer communities is not disproportionately influenced. Subsidies for recipients of the Supplemental Nutrition Assistance Program, such as that demonstrated in the Healthy Incentives Pilot [10], may be one method to promote more widespread access to produce an antibiotic-free product.

The AMA, Infectious Diseases Society of America, Centers for Disease Control and Prevention, and World Health Organization have issued statements, guidelines, and policies [11] in efforts to reduce use of antibiotics in animals intended for human consumption in the absence of therapeutic need. Some policies, however, contain major loopholes that allow producers to use antibiotics routinely, claiming therapeutic indication even when an indication does not exist. Broader policy reform is needed to address the routine use of antibiotics in livestock. The European Union has successfully instituted policies banning the routine use of antibiotics in animal feed, paving the way for other nations to follow in its lead. The United States should also forbid the use of antibiotics in animal agriculture for purposes other than controlling or treating veterinariandiagnosed disease. Antibiotics are sometimes indicated to treat infections in animals; however its routine prophylactic use is irresponsible because it contributes to AMR - a major global health threat. Given the seemingly impossible challenges in combating the public health crisis of AMR, the recommendation to stand against the routine use of prophylactic antibiotics in agriculture is a highly feasible strategy that is already making a difference.

Antibiotics save millions of human lives each year. As clinicians, we should unite around the proper stewardship of antibiotics in society, urging narrower indications both in patients and animals. Compared with the medical community's longstanding recommendation to eat low-fat foods, the recommendation to eat antibiotic-free foods is supported by data and is a far more urgent matter. This urgency is increasingly relevant to doctors around the world because of the global spread of western factory farming techniques that 
use mass antibiotics. AMR is a threat greater in magnitude than that of Zika and Ebola combined. On the basis of the public health threat of AMR, the routine use of antibiotics in farm animals for growth promotion or disease prevention should be banned worldwide. Physician-led health advocacy of antibiotic-free foods can be a powerful strategy in combatting this growing threat to patients.

\section{References}

1. Jukes TH, Stokstad E, Tayloe R, Cunha T, Edwards H, Meadows G. Growth-promoting effect of aureomycin on pigs. Arch Biochem. 1950;26:324-5.

2. Moore P, Evenson A, Luckey T, McCoy E, Elvehjem C, Hart E. Use of sulfasuxidine, streptothricin, and streptomycin in nutritional studies with the chick. J Biol Chem. 1946;165:437-41.

3. Table 3. Certified organic and total U.S. acreage, selected crops and livestock, 1995-2011. https://www.ers.usda.gov/webdocs/Da taFiles/Organic_Production_18002/CertifiedandtotalUSacrea geselectedcropslivestock.xls? $v=41571$. 2016. Accessed 17 March 2017.

4. FDA annual summary report on antimicrobials sold or distributed in 2015 for use in food-producing animals. https://www.fda.gov/
AnimalVeterinary/NewsEvents/CVMUpdates/ucm534244.htm. 2016. Accessed 17 March 2017.

5. Animal Health Institute. Fact or fiction: common antibiotic myths. http://www.ahi.org/issues-advocacy/animal-antibiotics/fact-orfiction-common-antibiotic-myths/. 2017. Accessed 17 Mar 2017.

6. Silbergeld EK, Graham J, Price LB. Industrial food animal production, antimicrobial resistance, and human health. Annu Rev Public Health. 2008;29:151-69.

7. Smith KE, Besser JM, Hedberg CW, et al. Quinolone-resistant Campylobacter jejuni infections in Minnesota, 1992-1998. New Engl J Med. 1999;340:1525-32.

8. Iovine NM, Blaser MJ. Antibiotics in animal feed and spread of resistant Campylobacter from poultry to humans. Emerg Infect Dis. 2004;10:1158.

9. Sneeringer S, MacDonald J, Key N, McBride W, Mathews K. Economics of Antibiotic Use in U.S. Livestock Production. USDA Economic Research Service; 2015.

10. US Department of Agriculture. Evaluation of the healthy incentives pilot (HIP) final report-summary. https://www.fns.usda. gov/sites/default/files/HIP-Final-Summary.pdf. 2014. Accessed 21 July 2017.

11. Food and Drug Administration. Guidance for Industy \# 213. New Animal Drugs and New Animal Drug Combination Products Administered in or on Medicated Feed or Drinking Water of Food Producing Animals: Recommendations for Drug Sponsors for Voluntarily Aligning Product Use Conditions with GFI \#209 (2012). 